\title{
Diversidad y distribución de peces y su relación con variables ambientales, en el sur del Golfo de México
}

\author{
Daniel Torruco ${ }^{1}$, Alicia González-Solis ${ }^{1} \&$ Ángel D. Torruco-González ${ }^{2}$ \\ 1. Centro de Investigaciones y de Estudios Avanzados del IPN Unidad Mérida, Km 6 Antigua carretera a Progreso. AP. \\ 79, Cordemex,97310 Mérida, Yucatán; dantor6660@gmail.com, aligonzalezsol@gmail.com \\ 2. Universidad Anáhuac-Mayab. Carretera Mérida-Progreso Km 15.5 AP 96, Cordemex, 97130 Mérida, Yucatán; \\ angel.11.daniel@gmail.com
}

Recibido 18-V-2017. Corregido 26-X-2017. Aceptado 25-XI-2017.

\begin{abstract}
Fish diversity, distribution and their relationship with environmental variables in Southern Gulf of Mexico. The Campeche Sound has a high heterogeneity of habitats, great fish species diversity, and a strong presence of oil and fishing industries. Our objective was to obtain updated information on a complete local fish species spatial distribution and their relationship with environmental elements, this will improve the baseline to evaluate the potential impact of future oil and fishing developments. For this, our approach used a database obtained in 2010, with a capture made in 54 sites with standard shrimp fishing trawls in the Campeche Sound. We collected a total of 33315 fish, of 80 families, 138 genera and 193 species, that corresponded to 2502438 $\mathrm{kg}$. The fish classes Chondrichthyes and Actinopterygii predominated. A total of 17 were dominant, associated to sandy and muddy bottoms, although were species of estuarine environments, reef and pelagic habitats. Averaged diversity (H') was of 3.2 bits/individuals. In decreasing diversity order, the common fish belong to Perciformes (84 species), Pleuronectiformes (23), Scorpaeniformes (15) and Tetraodontiformes (14); in the same way, the higher richness families were Sciaenidae (15 species), Paralichthyidae (12), Carangidae (10), and Triglidae and Synodontidae (7). Most common genuses were Prionotus (6 species), Citharichthys and Sphoeroides (4 each). Per each station, richness varied between 3 and 56 species, with an average weight of $46 \mathrm{~kg}$. Fish assemblage composition was different among habitats and areas, with divergent management strategies. Landscape analysis defines nodules of high density and diversity that corresponded to river mouths, lagoons and reef areas fish assemblages. Of the relation between variables and community descriptors, only the organic carbon was significant for both abundance and biomass, the REDOX for the species richness and the other variables were not important. Rev. Biol. Trop. 66(1): 438-456. Epub 2018 March 01.
\end{abstract}

Key words: ictiofauna; Campeche Sound; Gulf of Mexico; shrimp trawl.

Las áreas marino-costeras proporcionan al ser humano recursos naturales y servicios ambientales importantes, además de sus valores estéticos y recreativos. En México, son áreas de extracción de petróleo, gas y sal; por otro lado, la flora y fauna son también susceptibles de explotación y al mismo tiempo mantienen la diversidad del patrimonio genético del país (PNUMA, 2003). La zona sur del Golfo de México (Sonda de Campeche) es considerada como una de las regiones de gran importancia por su alto potencial biótico y de recursos naturales y es una de las más estudiadas por la diversidad de especies, tanto de vertebrados como de invertebrados, la heterogeneidad de hábitats, la expansión de la industria pesquera y portuaria, y por la explotación petrolera (García-Cuellar, Arreguín-Sánchez, Hernández, \& Lluch-Cota, 2004). Esta zona presenta una fuerte interacción con cuerpos lagunares, que conforman un complejo intercambio de masas de agua y en consecuencia, la organización de las especies está ligada a procesos deltaicos, estuarinos y oceanográficos. 
Los peces constituyen un elemento importante en las investigaciones de la zona, ya sea por su valor económico o por su valor ecológico; sin embargo, dada la creciente pérdida de ambientes costeros y marinos, se busca contar con inventarios actualizados y completos de la ictiofauna en todas las costas del país. Esta información es necesaria para la conservación de la biodiversidad, y requiere de un entendimiento profundo para que sea un elemento importante en el manejo y administración de los recursos naturales. Al considerar los argumentos anteriores, el presente trabajo buscó discernir cuáles variables afectan la abundancia, riqueza y distribución de los peces demersales, para que sirva como una línea base hacia los cambios o contingencias que pudieran presentarse en la zona.

\section{MATERIALES Y MÉTODOS}

El área de estudio comprendió la porción sur del Golfo de México, ubicada entre $\operatorname{los} 22^{\circ} 30^{\prime}-18^{\circ} 26^{\prime} \mathrm{N}-95^{\circ} 44^{\prime}-90^{\circ} 00^{\prime} \mathrm{W}$, en un polígono que abarcó el área costera y oceánica desde Alvarado, Veracruz, la plataforma continental de los estados de Tabasco y Campeche, hasta la Laguna de Celestún en Yucatán, y comprendió la bahía de Campeche y parte de la región suroeste del Banco de Campeche. El área fue muestreada mediante dos cruceros, ambos en la época de nortes (septiembreoctubre-noviembre) en el año 2010, y cubrió 54 sitios donde fue exitosa la colecta, mediante arrastre comercial de la ictiofauna bentodemersal (Fig. 1).

Los elementos registrados del ambiente se basaron en los componentes del fondo, dado que la ictiofauna bento-demersal está en íntimo contacto con este estrato. Para el sedimento $(0-10 \mathrm{~cm})$, las muestras se recolectaron con un nucleador de caja tipo Hessler_Sandia MK-II de 40 x $40 \mathrm{~cm}$ y una draga tipo SmithMacIntyre. La granulometría de los sedimentos se analizó suspendiendo una muestra (previamente secada en liofilizadora, durante $24 \mathrm{~h}$ ) en

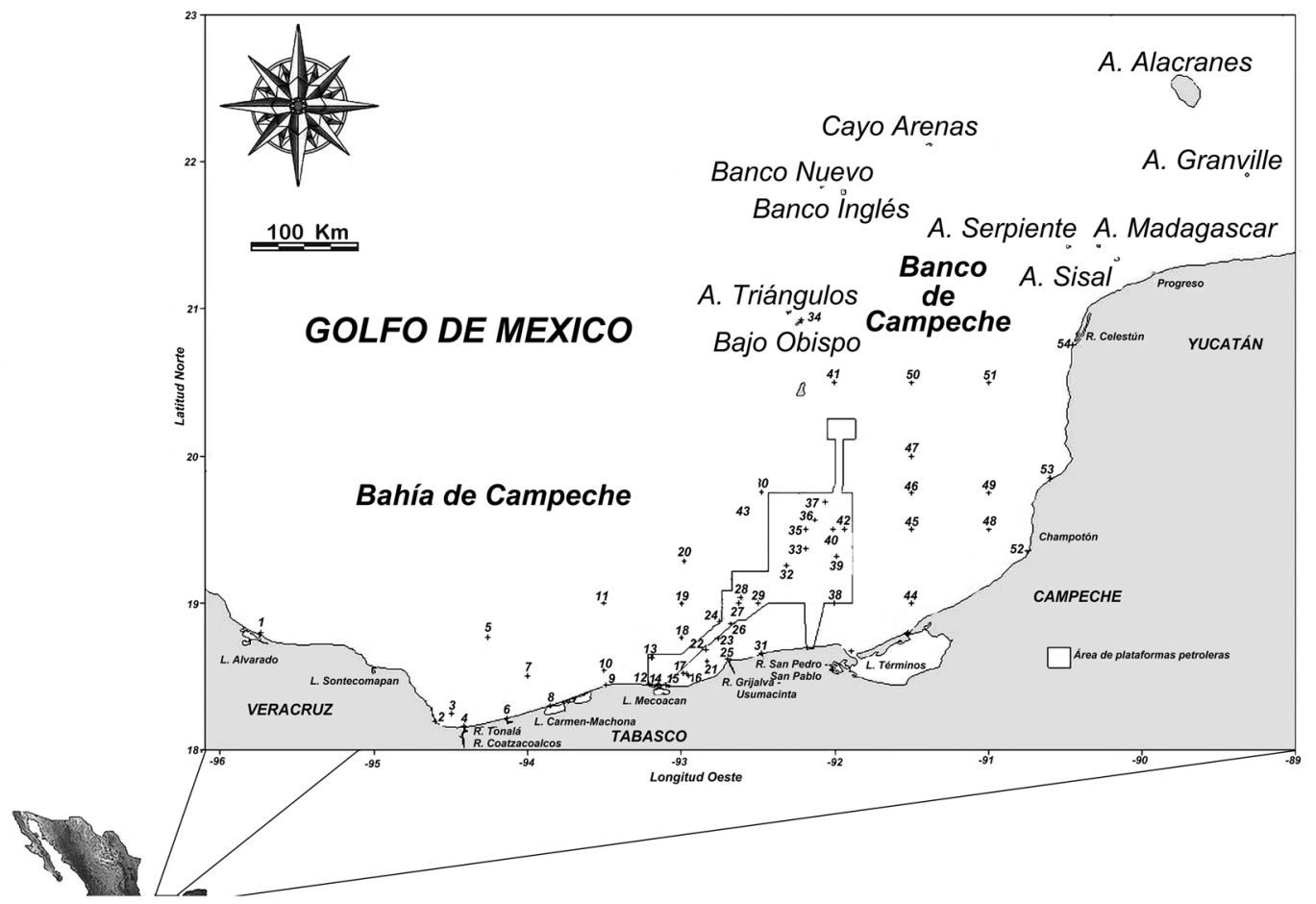

Fig. 1. Localización geográfica de los sitios de muestreo.

Fig. 1. Geographical location of the sampling sites. 
agua con surfactante y tomando muestras con pipeta a diferentes tiempos para luego estimar el tamaño de grano con la ley de sedimentación. Paralelamente, se hicieron mediciones de densidad con hidrómetro para corroborar resultados. Para la cuantificación y clasificación de las arenas, se usaron tamices de los números de malla: 400, 60, 40, 30, 20 y 10 todos ellos de la marca Davi (Buchanan, 1984). El contenido de carbono orgánico se cuantificó usando la técnica de oxidación con dicromato de potasio en medio ácido y titulación del exceso de oxidante con sulfato ferroso con difenilamina como indicador (Buchanan, 1984). El potencial Redox y el $\mathrm{H}_{2} \mathrm{~S}$ se midieron directamente con los electrodos respectivos y un potenciómetro, las mediciones se hicieron a $5 \mathrm{~cm}$ de profundidad directamente en la caja del nucleador (Bricker, 1982; Brassard, 1997).

Para la captura de los peces, se utilizaron redes camaroneras comerciales (60 pies de longitud y con luz de malla de 11/4 de pulgada), con lances de 30 minutos por cada estación, a una velocidad promedio de dos nudos, de acuerdo a la hora de llegada a cada punto el arrastre se realizó de día o de noche, en todos los casos se recorrió una distancia de una milla aproximadamente, las profundidades de colecta cubrieron un intervalo de 19 hasta los $600 \mathrm{~m}$. Todos los organismos capturados fueron congelados y trasladados al laboratorio, donde fueron lavados, separados, pesados, identificados y clasificados con el uso de guías de identificación y literatura apropiada (Dickson \& Moore, 1977; Sánchez- Gil et al., 1981; Aguirre-León \& Yáñez-Arancibia, 1986; Nelson, 1994, 2006; Torres \& Orozco, 1991; Lozano et al., 1993; Castro-Aguirre \& Espinoza-Pérez, 1996; Lavett, 1997; Espinoza-Pérez, Fuentes-Mata, Gaspar-Dillanes, \& Arenas, 1998; MacEachran \& Fechhelm, 1988a, 1988b; Carpenter, 2002a, 2002b, 2002c; Powell, Headrich, \& McEachran, 2003; Bumes-Romo, 2009)

Todos los datos fueron transformados (raíz-raíz) y procesados estadísticamente mediante análisis mono y multifactorial [análisis de dominancia (IVI), riqueza y diversidad de especies (índice de Shannon), equidad, análisis de agrupamiento (índice de Bray-Curtis), escaladores multidimensionales (ACP), análisis paisajístico y análisis de contenido de información $\left(\mathrm{CI}_{2}\right)$ ] con lo cual se caracterizaron las relaciones entre la estructura comunitaria y los factores abióticos (Pielou, 1978; Orlocí, 1978).

A partir de la determinación de cada punto muestral con los atributos comunitarios de la fauna, se formaron mapas temáticos digitales, que representaron los valores en el espacio, cuya variación es explicada al menos en forma parcial, utilizando funciones de correlación espacial midiendo la semivarianza entre datos separados por distancias diferentes mediante la función Y(h) (Modificada de Rossi, Mulla, Joumel \& Franz, 1992) la cual guarda la siguiente forma:

$$
\gamma(h)=\frac{\sum_{i=1}^{N(h)}\left(X_{i}-X_{i+1}\right)^{2}}{2 N(h)}
$$

Donde:

$\mathrm{Xi}$ y $\mathrm{Xi}+1=$ Son medidas del atributo comunitario en el punto i y en el punto $\mathrm{i}+1$.

$\mathrm{N}(\mathrm{h})=$ Número de puntos en la red de puntos muestrales.

Con estos datos se pudo diagramar isolíneas de altas y bajas densidades para cada uno de los elementos considerados, lo que representa un mapa bidimensional de la distribución espacial de los descriptores.

La matriz binaria de especies y sitios, fue analizada bajo el criterio del contenido de información de orden $2\left(\mathrm{CI}_{2}\right)$ en un proceso divisivo monotético, para identificar las especies discriminantes de cada sitio, a partir de la forma modificada de Ezcurra y Equihua (1984) que es la siguiente:

$$
I=2 s n \log n-n \sum_{i=1}^{s} \log \left[a^{2}+\left(n-a_{i}\right)^{2}\right]
$$

Donde:

$\mathrm{s}$ - número de descriptores

n - número de objetos

$\mathrm{a}_{1}$ - número de objetos en los que está presente el descriptor $\mathrm{i}$

Por último, para conocer la influencia de los factores del ambiente y sobre todo en el 
sentido de su importancia en un programa de monitoreo, para detectar cambios en la biota versus concentraciones de elementos presentes en el medio, se utilizó un análisis de regresión múltiple paso a paso con variables ambientales, bajo las siguientes consideraciones: Identificar si algún macro-descriptor comunitario tenía relación con algunas de las variables determinadas, asumiendo que no existe interdependencia entre las variables y que cumplen condiciones de normalidad.

Para este caso, los macrodescriptores comunitarios seleccionados fueron: riqueza de especies (S), diversidad (H'), abundancia (A) y biomasa (B). Las variables ambientales independientes, se seleccionaron entre 134; valores de hidrocarburos, granulometría y parámetros físicos y químicos del sedimento, bromatología del sedimento y plaguicidas. De ellas se escogieron las 19 más relevantes que tuvieran alguna influencia sobre la fauna demersal $\mathrm{y}$ de fondo, con las cuales se realizaron las correlaciones correspondientes. El criterio utilizado para analizar la relación del parámetro con los componentes comunitarios fue la significancia estadística.

\section{RESULTADOS}

Durante las campañas se capturaron un total de 33315 individuos que correspondieron a dos clases (Chondrichthyes y Actinopterygii), 24 órdenes, 80 familias, 138 géneros y 193 especies (Cuadro 1). El orden Perciformes fue el más diverso con 30 familias, 57 géneros y 84 especies. Seguidos por Pleuronectiformes (lenguados) con 23 especies, los Scorpaeniformes, con 15 y finalmente los Tetraodontiformes con 14. Las familias con mayor riqueza fueron Sciaenidae con 15 especies, Paralichthyidae con 12, Carangidae 10, y Triglidae y Synodontidae con siete cada una. En relación a los géneros, Prionotus Lacepède, 1801 con seis especies fue el mejor representado. Seguido por Citharichthys Bleeker, 1862 y Sphoeroides Lacepède, 1800 con cuatro cada uno.

En el Cuadro 2 se muestran las estaciones importantes por su gran abundancia (> a 1000 ejemplares) y biomasa ( $>$ a $100 \mathrm{~kg}$ ) o por lo escaso tanto de abundancia ( $<$ de 100 ejemplares) como de biomasa ( $<$ a $10 \mathrm{~kg}$ ); no obstante, el promedio de abundancia por estación fue de 617 individuos, mientras que la biomasa tuvo una variación de 1 a $212 \mathrm{~kg}$, con un promedio por estación de $46 \mathrm{~kg}$. Los ejemplares colectados fueron juveniles, preadultos y adultos. La riqueza de especies por estación tuvo una variación de tres a 56 especies.

El IVI (Índice del Valor de Importancia), detectó 17 especies dominantes debido a su amplia distribución, representan una considerable abundancia, una biomasa elevada y una frecuencia alta (Cuadro 3). Algunas de

\section{CUADRO 1}

Órdenes y número de familias, géneros y especies comprendidos en las campañas de muestreo

TABLE 1

Orders and families, genera and species number in the sampling campaigns

\begin{tabular}{|c|c|c|c|}
\hline ÓRDEN & Familia & Género & Especie \\
\hline Chimaeriniformes & 1 & 1 & 1 \\
\hline Charcharhiniformes & 2 & 2 & 2 \\
\hline Torpediniformes & 1 & 1 & 1 \\
\hline Rajiformes & 3 & 5 & 5 \\
\hline Myliobatiformes & 5 & 5 & 6 \\
\hline Elopiformes & 1 & 1 & 1 \\
\hline Albuliformes & 1 & 1 & 1 \\
\hline Anguiliformes & 4 & 5 & 5 \\
\hline Cupleiformes & 2 & 6 & 6 \\
\hline Sluriformes & 1 & 2 & 2 \\
\hline Ateleopidiformes & 1 & 1 & 1 \\
\hline Aulopiformes & 2 & 5 & 8 \\
\hline Polymixiiformes & 1 & 1 & 1 \\
\hline Ophidiiformes & 1 & 3 & 3 \\
\hline Gadiformes & 3 & 3 & 3 \\
\hline Batrachoidiformes & 1 & 1 & 1 \\
\hline Lophidiiformes & 3 & 5 & 7 \\
\hline Antheriniformes & 1 & 1 & 1 \\
\hline Zeiformes & 1 & 1 & 1 \\
\hline Gasterosteiformes & 1 & 1 & 1 \\
\hline Scorpaeniformes & 4 & 7 & 15 \\
\hline Perciformes & 30 & 57 & 84 \\
\hline Pleuronectiformes & 5 & 14 & 23 \\
\hline Tetraodontiformes & 5 & 9 & 14 \\
\hline TOTAL & 80 & 138 & 193 \\
\hline
\end{tabular}


CUADRO 2

Sitios de mayor y menor abundancia y peso

TABLE 2

Greater and lesser abundance and weight sites

$\begin{array}{cccccccc}\text { Estaciones } & \begin{array}{c}\text { No. de ejemplares } \\ >1000\end{array} & \text { Estaciones } & \begin{array}{c}\text { No. de ejemplares } \\ <100\end{array} & \text { Estaciones } & \text { Biomasa }>100 \text { kg. } & \text { Estaciones } & \text { Biomasa }<10 \mathrm{~kg} \\ 17 & 3766 & 26 & 98 & 53 & 212 & 48 & 7 \\ 9 & 2499 & 46 & 86 & 9 & 196 & 3 & 5 \\ 1 & 1906 & 11 & 80 & 39 & 132 & 7 & 5 \\ 39 & 1606 & 22 & 73 & 54 & 122 & 22 & 5 \\ 36 & 1590 & 24 & 72 & 43 & 116 & 23 & 5 \\ 40 & 1478 & & & 1 & 102 & 5 & 4 \\ 31 & 1420 & & & & & & \\ 43 & 1280 & & & & & & \\ 35 & 1261 & & & & & & \\ 4\end{array}$

CUADRO 3

Especies dominantes por el índice de valor de importancia

TABLE 3

Dominant species by the index's value of importance

\begin{tabular}{lcccc}
\multicolumn{1}{c}{ Especie } & Abundancia relativa (\%) & Biomasa relativa (\%) & Frecuencia & IVI (\%) \\
Eucinostomus gula & 12.01 & 4.72 & 35 & 19.67 \\
Ariopsis felis & 6.32 & 9.43 & 31 & 18.35 \\
Chloroscombrus chrysurus & 12.89 & 2.22 & 22 & 16.95 \\
Syacium gunteri & 6.40 & 1.49 & 38 & 11.08 \\
Synodus foetens & 2.94 & 3.98 & 24 & 10.19 \\
Cynoscion nothus & 3.86 & 3.71 & 31 & 9.58 \\
Lutjanus synagris & 1.76 & 4.58 & 15 & 8.95 \\
Stellifer colonensis & 5.57 & 1.24 & 20 & 8.08 \\
Bagre marinus & 2.88 & 3.12 & 18 & 7.68 \\
Conodon nobilis & 1.90 & 3.60 & 38 & 7.01 \\
Prionotus paralatus & 1.93 & 1.46 & 14 & 6.58 \\
Lutjanus campechanus & 1.45 & 3.22 & 20 & 5.85 \\
Upeneus parvus & 2.62 & 1.41 & 12 & 5.71 \\
Micropogonias undulatus & 0.69 & 3.50 & 19 & 5.20 \\
Diapterus auratus & 2.22 & 1.20 & 20 & 5.02 \\
Diapterus rhombeus & 1.86 & 1.02 & 20 & 4.56 \\
Narcine brasiliensis & 0.58 & 2.03 & & 4.29 \\
\hline
\end{tabular}

estas especies requieren de un conocimiento más profundo sobre su biología y ecología, ya que no son explotadas comercialmente; pero representan un potencial recurso que puede ser aprovechado en la medida que se vaya generando más información sobre ellas. De estas especies, las que se explotan de manera comercial actualmente son Lutjanus campechanus (Poey, 1860) y L. synagris (Linnaeus, 1758), Ariopsis felis (Linnaeus, 1766), Bagre marinus (Mitchill, 1815) y Micropogonias undulatus (Linnaeus, 1766) 
Los mayores valores del índice de Shannon-Wiener se presentaron entre los cuatro bits/ind. mientras que el mínimo reportado fue de 1.0 bits/ind. La equidad más alta superó los 0.9 y la menor fue de 0.24 . En el cuadro 4 se presentan los valores de riqueza de especies, diversidad y equidad.
El análisis de agrupamiento de los sitios de colecta de acuerdo al índice de Bray-Curtis, para la abundancia y la biomasa, se realizó a un nivel de corte de $75 \%$ de disimilitud. Con la abundancia se formaron cuatro grupos: el I se integró por 16 estaciones que presentaron una alta abundancia (mayores a 700 organismos

CUADRO 4

Riqueza de especies, diversidad y equitatividad en las estaciones de colecta de la fauna íctica

TABLE 4

Species richness, diversity and evenness in the fish fauna collection stations

\begin{tabular}{|c|c|c|c|c|c|}
\hline Clave sitio* & Coor & nadas & S & $\mathrm{H}^{\prime}$ & $J^{\prime}$ \\
\hline 1 & $1847^{\prime} 31^{\prime \prime}$ & -95 44’53”' & 37 & 3.824 & 0.734 \\
\hline 2 & $1811^{\prime} 42^{\prime \prime}$ & $-9435^{\prime} 58^{\prime \prime}$ & 34 & 4.280 & 0.841 \\
\hline 3 & $1815^{\prime} 00^{\prime \prime}$ & -94 30’00" & 15 & 3.466 & 0.887 \\
\hline 4 & $1809^{\prime} 30$ " & $-9424{ }^{\prime} 42^{\prime \prime}$ & 41 & 3.950 & 0.737 \\
\hline 5 & $1845^{\prime} 43^{\prime \prime}$ & $-9415^{\prime} 31^{\prime \prime}$ & 12 & 3.087 & 0.861 \\
\hline 6 & $1818^{\prime} 45^{\prime \prime}$ & $-9400 ’ 00$ ' & 23 & 3.029 & 0.670 \\
\hline 7 & $1830^{\prime} 00^{\prime \prime}$ & $-9400 ’ 00$ ' & 27 & 3.995 & 0.840 \\
\hline 8 & $1818^{\prime} 09^{\prime \prime}$ & -93 50’53” & 25 & 3.407 & 0.734 \\
\hline 9 & $1825^{\prime} 00^{\prime \prime}$ & $-9329^{\prime} 13^{\prime \prime}$ & 56 & 4.572 & 0.787 \\
\hline 10 & $1830^{\prime} 00^{\prime \prime}$ & -93 30’00" & 31 & 3.683 & 0.743 \\
\hline 11 & 19 00’00" & -93 30’00" & 15 & 3.771 & 0.965 \\
\hline 12 & $1826^{\prime} 57^{\prime \prime}$ & $-9312^{\prime} 26^{\prime \prime}$ & 39 & 4.398 & 0.832 \\
\hline 13 & $1837^{\prime} 27.41 "$ & $-9311^{\prime} 25.78^{\prime \prime}$ & 29 & 3.790 & 0.780 \\
\hline 14 & $1828^{\prime} 59.17^{\prime \prime}$ & $-9259^{\prime} 12.66^{\prime \prime}$ & 22 & 3.785 & 0.849 \\
\hline 15 & $1826^{\prime} 30^{\prime \prime}$ & $-9308^{\prime} 41^{\prime \prime}$ & 31 & 3.259 & 0.658 \\
\hline 16 & $1826^{\prime} 28^{\prime \prime}$ & $-9305{ }^{\prime} 27^{\prime \prime}$ & 30 & 3.526 & 0.719 \\
\hline 17 & $1830^{\prime} 00^{\prime \prime}$ & $-9300 ’ 00 "$ & 23 & 1.093 & 0.242 \\
\hline 18 & $1845^{\prime} 00^{\prime \prime}$ & $-9300 ’ 00 "$ & 12 & 2.133 & 0.595 \\
\hline 19 & 19 00’00”' & -93 00’00” & 26 & 3.203 & 0.681 \\
\hline 20 & 19 17'06.15" & $-9258^{\prime} 45.05 "$ & 23 & 3.246 & 0.717 \\
\hline 21 & $1837^{\prime} 36.54$ " & -92 51'54.33”' & 36 & 4.254 & 0.823 \\
\hline 22 & $1845^{\prime} 36.01 "$ & $-9245^{\prime} 32.33 ”$ & 17 & 3.478 & 0.851 \\
\hline 23 & $1851^{\prime} 38.38$ ' & $-9240^{\prime} 28.50 ”$ & 13 & 2.965 & 0.801 \\
\hline 24 & $1852^{\prime} 36.41^{\prime \prime}$ & $-9245^{\prime} 00.20^{\prime \prime}$ & 9 & 2.841 & 0.896 \\
\hline 25 & 18 36’39” & -92 41’46” & 17 & 2.241 & 0.548 \\
\hline 26 & 19 00’00” & $-9237^{\prime} 30^{\prime \prime}$ & 10 & 2.890 & 0.870 \\
\hline 27 & 1907 '30” & $-9233^{\prime} 45^{\prime \prime}$ & 14 & 3.193 & 0.839 \\
\hline 28 & 19 00’ 00” & -92 30’00” & 15 & 3.263 & 0.835 \\
\hline 29 & $1911 ' 15^{\prime \prime}$ & -92 30’00” & 13 & 3.119 & 0.843 \\
\hline 30 & $1930 ’ 00 ”$ & $-9230 ’ 00 ”$ & 18 & 3.112 & 0.746 \\
\hline 31 & 18 39'03" & -92 28’08" & 20 & 2.540 & 0.588 \\
\hline 32 & $1915^{\prime} 00^{\prime \prime}$ & $-9218^{\prime} 45^{\prime \prime}$ & 31 & 3.783 & 0.764 \\
\hline 33 & $1930 ’ 00 ”$ & $-9211 ' 15^{\prime \prime}$ & 26 & 3.045 & 0.648 \\
\hline 34 & $2055^{\prime} 22.79^{\prime \prime}$ & $-9213 ' 11.99 "$ & 3 & 1.023 & 0.645 \\
\hline 35 & $1926^{\prime} 15^{\prime \prime}$ & -9207 ’30” & 28 & 2.876 & 0.598 \\
\hline
\end{tabular}


CUADRO 4 (Continuación) / TABLE 4 (Continued)

\begin{tabular}{|c|c|c|c|c|c|}
\hline Clave sitio* & \multicolumn{2}{|c|}{ Coordenadas } & S & $\mathrm{H}^{\prime}$ & $\mathrm{J}^{\prime}$ \\
\hline 36 & $1933^{\prime} 45^{\prime \prime}$ & $-9207^{\prime} 30 ”$ & 22 & 2.352 & 0.527 \\
\hline 37 & $1941^{\prime} 15^{\prime \prime}$ & $-9203^{\prime} 45^{\prime \prime}$ & 16 & 3.494 & 0.874 \\
\hline 38 & 1900 '00" & -92 00’00" & 17 & 3.384 & 0.828 \\
\hline 39 & 19 18'46.44”' & $-9159 ’ 34.22 ”$ & 36 & 3.385 & 0.655 \\
\hline 40 & 19 30'00.10" & -92 00’38.91” & 18 & 1.989 & 0.477 \\
\hline 41 & 2030 '00” & -92 00’00” & 8 & 2.443 & 0.814 \\
\hline 42 & 19 30'00" & $-9156^{\prime} 15^{\prime \prime}$ & 12 & 2.774 & 0.774 \\
\hline 43 & 18 40' $17^{\prime \prime}$ & $-9153^{\prime} 36^{\prime \prime}$ & 31 & 3.534 & 0.713 \\
\hline 44 & 18 47'03' & $-9130^{\prime} 56^{\prime \prime}$ & 34 & 3.947 & 0.776 \\
\hline 45 & 1930 '00" & $-9130 ’ 00 "$ & 14 & 2.168 & 0.569 \\
\hline 46 & $1945^{\prime} 00^{\prime \prime}$ & $-9130 ’ 00 "$ & 8 & 2.769 & 0.923 \\
\hline 47 & $2000^{\prime} 00^{\prime \prime}$ & $-9130 ’ 00 "$ & 6 & 2.096 & 0.811 \\
\hline 48 & 20 30'00" & $-9130 ’ 00 "$ & 8 & 2.719 & 0.906 \\
\hline 49 & 1930 '00" & $-9100 ’ 00$ ' & 18 & 3.632 & 0.871 \\
\hline 50 & $1945^{\prime} 00^{\prime \prime}$ & $-9100 ’ 00 "$ & 15 & 3.414 & 0.874 \\
\hline 51 & $2030^{\prime} 00^{\prime \prime}$ & $-9100 ’ 00 "$ & 8 & 2.095 & 0.698 \\
\hline 52 & $1921^{\prime} 30$ " & $-9043^{\prime} 24^{\prime \prime}$ & 36 & 4.435 & 0.858 \\
\hline 53 & $1948^{\prime} 55^{\prime \prime}$ & -90 36’00” & 38 & 4.404 & 0.839 \\
\hline 54 & 20 44'53”' & -90 26’ 16" & 24 & 3.891 & 0.849 \\
\hline
\end{tabular}

*Los sitios están identificados en la Fig. 1.

hasta 200) de organismos; el grupo II se conformó por 28 sitios de abundancias medias y bajas desde los 600 hasta los 80 organismos; el grupo III se constituyó por cinco localidades que compartieron una abundancia de organismos media y baja desde los 800 organismos hasta los 60; el grupo IV se formó por cinco sitios con abundancias altas que rebasaron los 1000 organismos (Fig. 2A).

La biomasa exhibió también cuatro grupos: el grupo I involucró sitios con alta biomasa $(>100 \mathrm{~kg})$. El segundo grupo incluyó a nueve localidades con abundancia media (100 a $40 \mathrm{~kg}$ ). El grupo III agrupó 23 sitios todos ellos con biomasa baja (40 a $1 \mathrm{~kg}$ ). El último grupo (IV), integró una agrupación de cinco sitios también con biomasas bajas (desde 5 a $50 \mathrm{~kg}$, Fig. 2B).

El Análisis de Componentes Principales (ACP) indicó que la mayoría de las especies se ordenaron muy cercanas al origen del espacio n-dimensional conformado un grupo compacto, aun cuando el primer eje fue el más importante, ya que explica el $68.85 \%$ de la varianza. Tres especies se alejan de este esquema: Citharichthys dinoceros Goode \& Bean, 1886, Evoxymetopon taeniatus Gill, 1863, y Stenotomus caprinus Jordsan \& Gilbert, 1882 especies que aunque se acercaron en un eje al origen, la disposición para los otros ejes los alejaron del mismo; las dos primeras se caracterizaron por ser especies de abundancia baja y muy poco frecuente, $S$. caprinus fue de abundancia media y frecuente, por ello se relacionó más cercana a Bairdiella ronchus (Cuvier, 1830), Bairdiella chrysoura (Lacepède, 1802), Cyclopsetta chittendeni Bean, 1895, Hoplunnis diomediana Goode \& Bean, 1896 y Dactylopterus volitans (Linnaeus, 1758), que además marcaron un gradiente que salió del origen y se elevó en el segundo eje de importancia (Fig. 3A).

El esquema que presentó la biomasa cambió formando dos grupos y varias especies separadas. El primer grupo se conformó por Bagre marinus, Ariopsis felis, Orthopristis chrysoptera (Linnaeus, 1766), Micropogonias undulatus y Archosargus probatocephalus (Walbaum, 1792), especies con una alta 


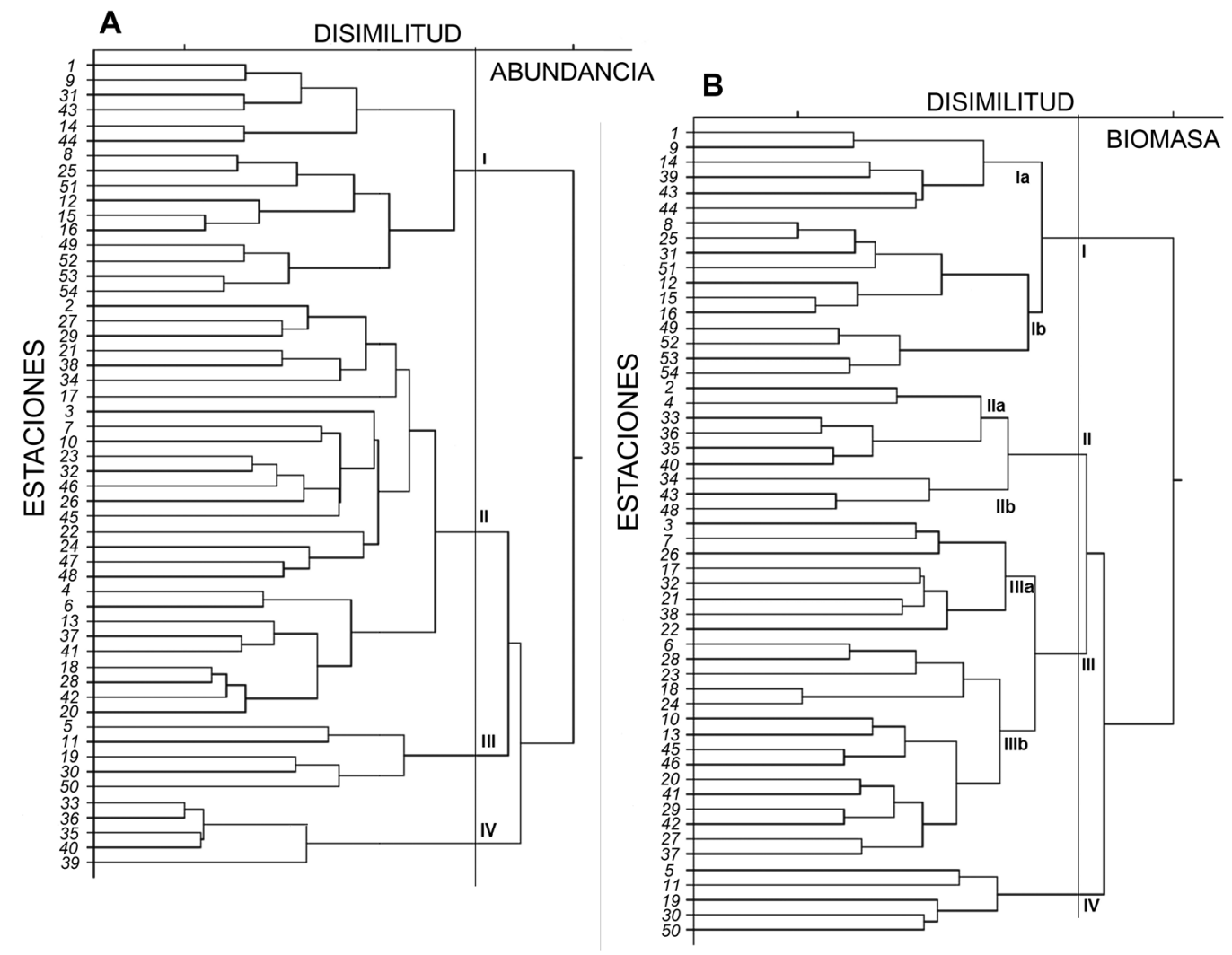

Fig. 2. Agrupamiento de estaciones en relación a la abundancia (A) y a la biomasa (B) de la ictiofauna de la Sonda de Campeche.

Fig. 2. Grouo sites in relation to the abundance (A) and biomass (B) of the Ichthyofauna of the Campeche Sound.

biomasa y muy frecuentes. El segundo grupo lo conformaron, la mayoría de las especies en un grupo compacto cercano al origen de los tres ejes, del cual se desprendieron como especies solitarias Conodon nobilis Linnaeus, 1758 y Umbrina coroides Cuvier, 1830, que, si bien fueron especies con alta biomasa, su frecuencia es media. Las especies solitarias como Cynoscion nothus Holbrook, 1848, Chaetodipterus faber, Eucinostomus gula Quoy \& Gaimard, 1824, y Haemulon plumierii Lacepède, 1801, fueron especies de biomasas altas y medias, pero con poca frecuencia. Hypanus sabinus Lesueur, 1824 presentó una alta biomasa, pero con una frecuencia reducida, es por ello que se separó de cualquier conglomerado. Lutjanus synagris fue una especie que presentó una alta biomasa y muy frecuente, lo que también la separó del grupo principal (Fig. 3B).
La abundancia de peces en los sitios presentó un patrón que formó tres agrupaciones y una separación de dos estaciones de abundancias altas ( 17 y 40 ). La primera congregó sitios con actividades petroleras, pero con abundancias altas. La segunda agrupó cuatro sitios cercanos a las desembocaduras de ríos y lagunas que registraron abundancias altas. La última agrupación reunió sitios cercanos al origen de los ejes y representaron abundancias de medias a bajas. La última reunió a las estaciones restantes muy cercanas al origen de los ejes, fue un grupo conformado con estaciones con abundancias medias a bajas; algunas marcan el final de un gradiente donde su máxima densidad se presentó cercana al origen (Fig. 3C)

Aun cuando los ejes del ACP explicaron solo el $48.17 \%$ de la varianza, las biomasas de las localidades presentaron un grupo definido 


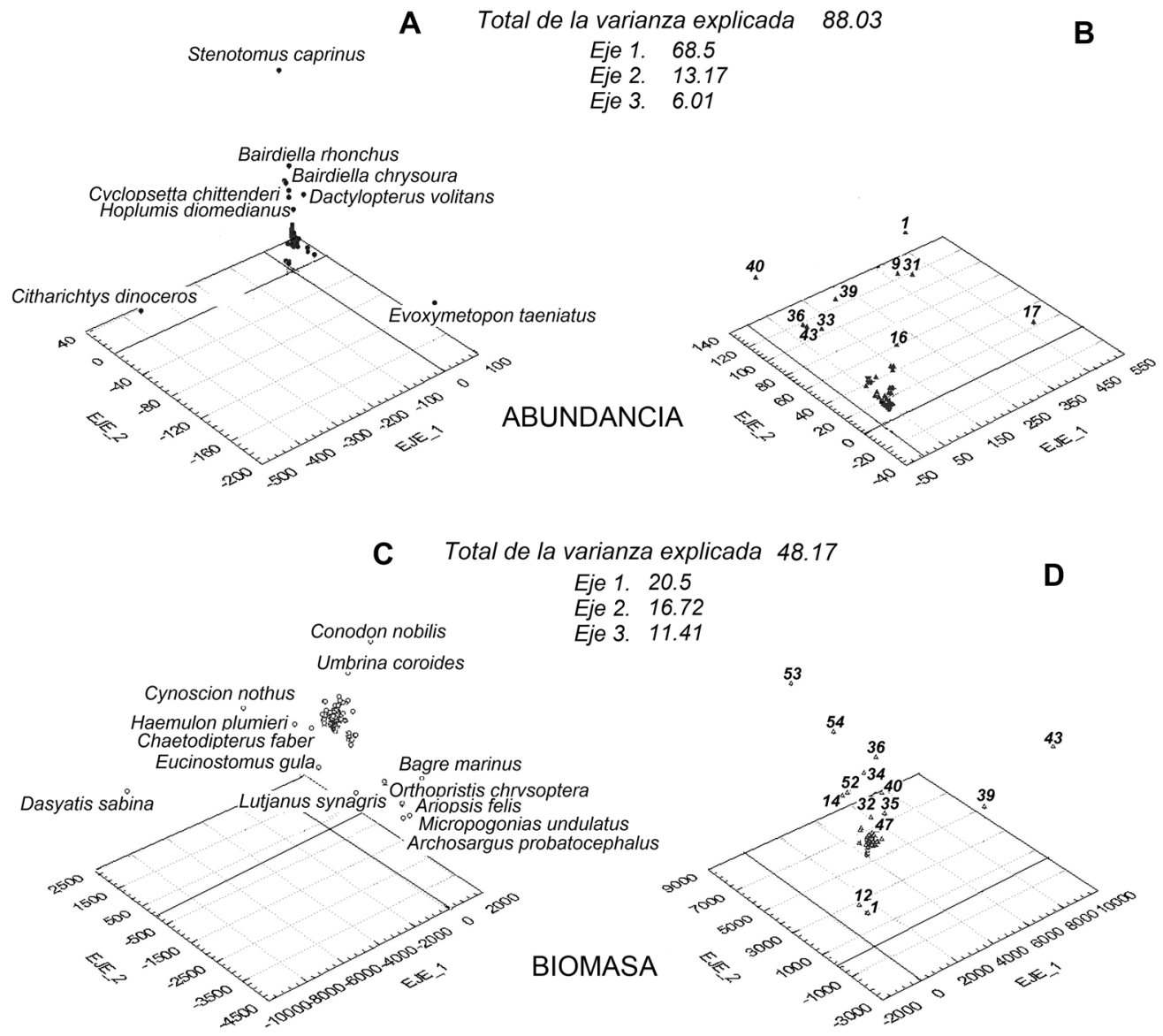

Fig. 3. Distribución espacial de las especies de peces (A, C) y los sitios de muestreo (B, D) en la Sonda de Campeche. Fig. 3. Spatial distribution of fish species (A, C) and sampling sites (B, D) in Campeche Sound.

que congregó a la mayoría de ellas; no obstante, se presentaron varios sitios como una nube muy cercana a este grupo, lo que puso de manifiesto su relación estrecha. Seis sitios se separaron de esta agrupación: 53, 54, 43, 39, 9 y 1 . Todas ellas fueron estaciones de gran biomasa, la mayoría cercanas a las desembocaduras de ríos y lagunas con excepción de la 39, que se ubicó cercana a lugares con actividad petrolera. En la nube de estaciones cercanas al conglomerado principal, se destacó la relación pareada de algunos sitios como es el caso de 52 y 14,36 y 34,40 y 32,35 y 47 , si bien la relación fue estrecha dada su cercanía, las características de cada estación fueron diferentes, el único denominador común fue que en cada par se presentó una estación cercana a actividades petroleras (Fig. 3D).

Para describir los patrones geoestadísticos de los descriptores de la ictiofauna, se siguió la misma secuencia donde se analizaron por separado cada uno de ellos.

La abundancia de la fauna de peces, presentó nódulos de alta densidad en las zonas costeras del área de muestreo, particularmente frente a las desembocaduras de las lagunas Mecoacan, Carmen-Machona y Alvarado (Fig. 4A). La biomasa de la ictiofauna también registró nódulos de alta densidad en la zona costera, de manera particular frente a las costas 

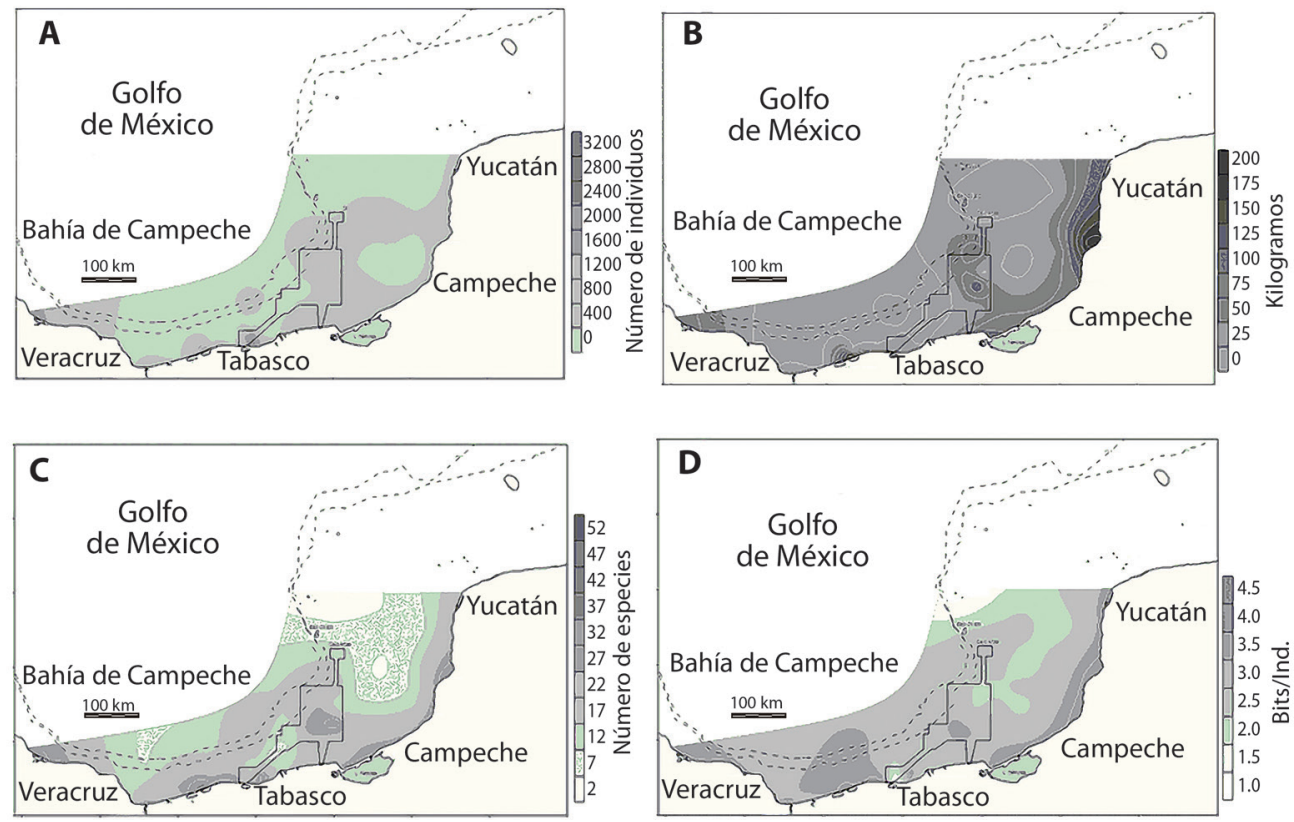

Fig. 4. Distribución de los parámetros comunitarios: abundancia (A), biomasa (B), riqueza de especies (C) y diversidad (D), de la fauna íctica de la Sonda de Campeche. Se muestran los altos nódulos en la porción costera cercana a las desembocaduras de los ríos/lagunas costeras y en las zonas de exclusión con plataformas petroleras.

Fig. 4. Community parameters distribution: abundance (A), biomass (B), species richness (C), and diversity (D), of the Campeche Sound fish fauna. High nodules in the portion coastal close to the rivers / coastal lagoons mouths and in them exclusion areas with platforms oil are shown.

de Campeche y en las cercanías de las lagunas Carmen-Machona, con valores cercanos a los $200 \mathrm{~kg}$. En las áreas de plataformas, también se presentaron nódulos con densidades de hasta $150 \mathrm{~kg}$ (Fig. 4B). El patrón espacial que mostró la riqueza de especies, propuso nódulos de altos valores (40-50 especies) cercanos a las lagunas costeras, donde se presentan los ambientes estuarinos que reúnen especies con tendencias eurihalinas y especies marinas; no obstante, también se presentaron valores altos en la zona de las plataformas petroleras (Fig. 4C). Los valores de diversidad se distribuyeron espacialmente de la siguiente manera: la zona cercana a la costa presentó altos valores, pero no se concentraron en ninguna área en particular, sino que se distribuyeron con cierta homogeneidad. Las zonas con menor contenido de información fueron las zonas más oceánicas. Esto indicó que se presenta un fuerte gradiente que va desde la zona costera (mayor diversidad ecológica), hasta la zona más alejada (menor diversidad ecológica) (Fig. 4D).

La clasificación divisiva monotética bajo el criterio del contenido de información de segundo orden dio como resultado un total de 24 especies discriminantes para los 54 sitios de registros de ictiofauna. El sitio y la especie que lo discriminó, así como el valor del $\mathrm{CI}_{2}$ se presentan en la figura 5. Las especies discriminantes más frecuentes fueron Syacium papillosum Linnaeus, 1758 y Trichiurus lepturus Linnaeus, 1758, seguidas de Upeneus parvus Poey, 1852 y Urobatis jamaicensis Cuvier, 1816. De acuerdo a las características de las localidades, 8 especies discriminaron a las cercanas a actividades petroleras; 3 discriminaron a filtraciones naturales de hidrocarburos, 16 discriminaron a sitios oceánicos y 7 a los cercanos a desembocaduras de ríos y lagunas. No obstante que 


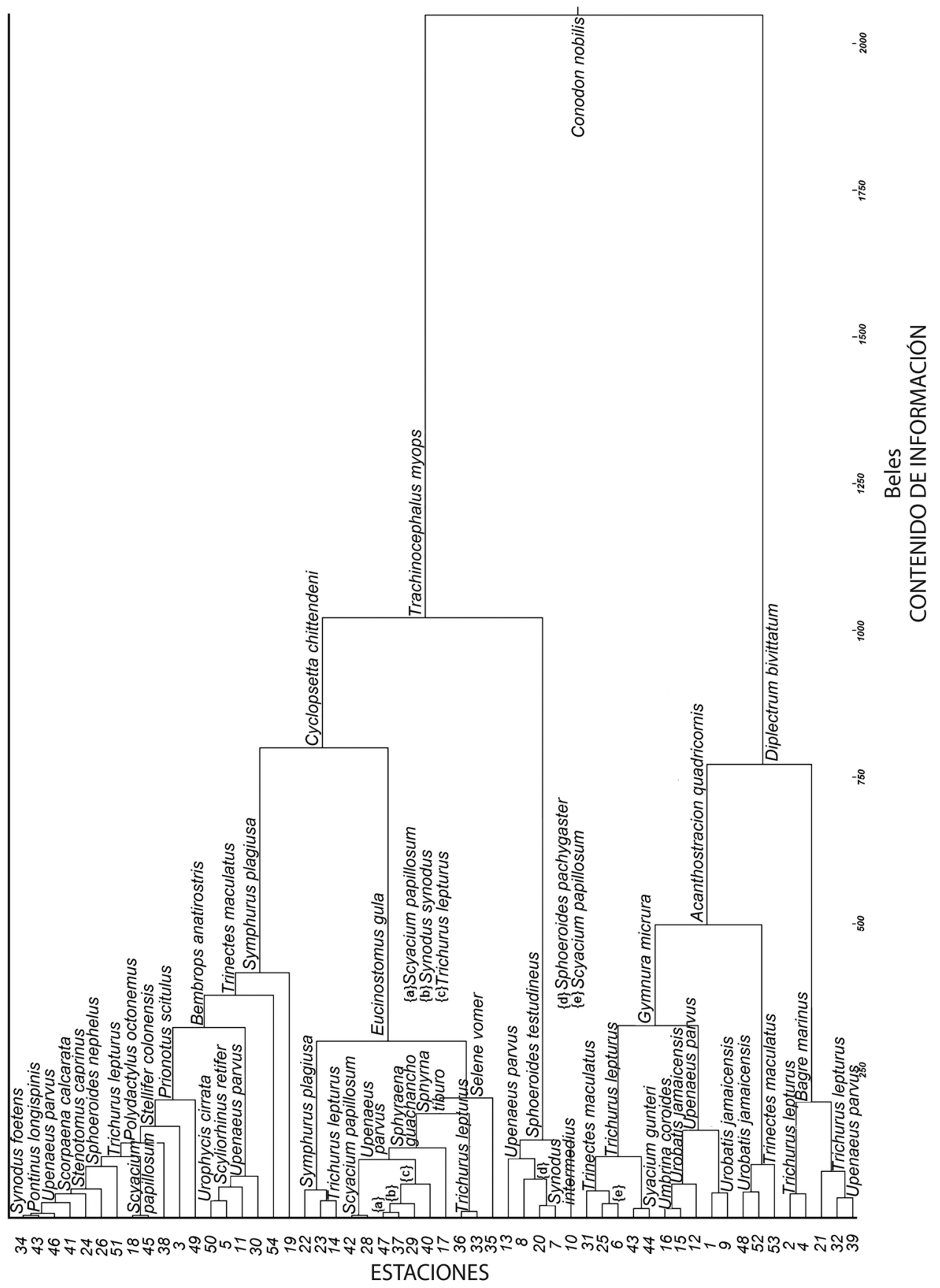

Fig. 5. Dendrograma que muestra las especies que discriminan cada sitio de muestreo con la presencia/ausencia e la fauna ictica de la Sonda de Campeche. Se muestra la caída de información y la especie responsable.

Fig. 5. Dendrogram showing the species that discriminate each sampling site with the fish fauna presence/absence in the Campeche Sound.Lack of information and the responsible species are shown. 
CUADRO 5

Valores de la regresión múltiple paso a paso de los parámetros comunitarios como variables dependientes versus parámetros del ambiente como variables independientes

TABLE 5

Values of stepwise multiple regression of community parameters as dependent variables versus environmental parameters as independent variables

\begin{tabular}{|c|c|c|c|c|c|c|c|c|c|}
\hline $\begin{array}{l}\text { No. de } \\
\text { casos }\end{array}$ & $\begin{array}{l}\text { No. de } \\
\text { pasos }\end{array}$ & $\begin{array}{c}\text { Variable } \\
\text { dependiente }\end{array}$ & $\begin{array}{c}\text { Variables ambientales } \\
\text { (independientes) y pesos Beta }\end{array}$ & $\begin{array}{c}\mathrm{R} \\
\text { múltiple }\end{array}$ & $\mathrm{R}^{2}$ & $\mathrm{~F}$ & $\mathrm{p}$ & Intercepto & $\begin{array}{l}\text { Error } \\
\text { Estándar }\end{array}$ \\
\hline 54 & 4 & Abundancia & $\begin{array}{l}\text { Carbono Orgánico }=. \mathbf{3 3 9} * \\
\text { Profundidad }=-.13\end{array}$ & .40 & .16 & 2.33 & .068 & 124.52 & 274.65 \\
\hline 54 & 6 & Biomasa & $\begin{array}{l}\text { Profundidad }=-.17 \\
\text { Carbono Orgánico }=. \mathbf{3 8 7} *\end{array}$ & .5 & .25 & 2.61 & .028 & 66782.27 & 21599.79 \\
\hline 54 & 4 & Riqueza de especies & $\begin{array}{l}\text { Redox }=. \mathbf{4 5 3} * \\
\text { Carbono Orgánico }=.317\end{array}$ & .55 & .31 & 5.57 & .0008 & 23.82 & 4.88 \\
\hline 54 & 3 & Diversidad & $\begin{array}{l}\text { Redox }=.324 \\
\text { Humedad }=-.35\end{array}$ & .53 & .28 & 6.53 & .0008 & 3.94 & .34 \\
\hline
\end{tabular}

*Variables estadísticamente significativas.

estas especies fueron las que, por su presencia o ausencia, finalmente separaron a las localidades, las responsables de las mayores caídas de información fueron: Conodon nobilis, que representó una caída de información total de 254.4 beles, Trachinocephalus myops Foster, 1801 con 76.5 beles, Diplectrum bivittatum Valenciennes, 1828 con 85.9 beles, Cyclopsetta chittendeni con 65.6 beles.

De las 19 variables ambientales incluidas en el modelo de regresión múltiple, solo dos tuvieron efecto significativo sobre las variables de respuesta (Cuadro 5). Con base en el coeficiente de regresión, la abundancia de los peces tiene cuatro variables importantes, de ellas, el carbono orgánico es el único que fue estadísticamente significativo. La biomasa presentó seis variables importantes, pero también sólo el carbono orgánico fue estadísticamente significativo; sin embargo, en ambos descriptores, la profundidad, sin ser un elemento significativo, influyó determinantemente. La riqueza de especies presentó cuatro variables importantes, sólo el REDOX fue estadísticamente significativo. La diversidad registró tres variables de importancia, pero ninguna fue estadísticamente significativa.

\section{DISCUSIÓN}

La riqueza de peces bentónicos y demersales para la parte sur del Golfo de México está integrada por 193 especies. Este valor registrado es alto si se compara con las 149 y 152 especies que reportaron Yáñez-Arancibia et al. (1985) y Yáñez-Arancibia y Sánchez-Gil (1986) respectivamente, para la Sonda de Campeche, la cual constituye una amplia región de la plataforma continental tropical frente a la Laguna de Términos.

Aunque de acuerdo con Yáñez-Arancibia y Sánchez-Gil (1986), la riqueza de especies podría integrarse por más de 270 especies en esta zona. Para la parte norte del Golfo, Powell, Headrich y McEachran (2003) reportaron 119 peces demersales, obteniendo una abundancia total de 1250 ejemplares provenientes de 31 sitios de muestreo, pero abarcando profundidades de más de $3000 \mathrm{~m}$. Estos autores concluyeron que la abundancia y la diversidad decrecen conforme aumenta la profundidad de muestreo. En ese contexto es probable que la riqueza en la parte sur del Golfo sea mayor que en la del norte, porque para esta campaña oceanográfica las profundidades no sobrepasaron los $600 \mathrm{~m}$. 
Por lo anterior, sería necesario y recomendable, muestrear a mayores profundidades, para poder hacer una comparación más real con la ictiofauna de la parte norte. Sin embargo, en el trabajo de Yáñez-Arancibia y Sánchez-Gil (1986) presentaron una abundancia total de más de 53700 ejemplares provenientes de 160 arrastres capturados en un periodo menor a un año con una profundidad máxima de $80 \mathrm{~m}$, y cubriendo un área menor a la del presente trabajo. En el presente trabajo, se presentan 33315 ejemplares en 54 estaciones ( $34 \%$ ), lo que puede ser indicativo de que la riqueza y abundancia del sur del Golfo de México puede ser aún mayor y se reitera la necesidad de realizar lances a mayores profundidades, para por lo menos alcanzar un poco más de $1000 \mathrm{~m}$, considerando que Yañez y Sánchez-Gil obtuvieron 152 especies y este trabajo 193.

Las causas posibles de estas diferencias en cuanto al número de especies pueden radicar en la cantidad de ejemplares recolectados, la cantidad y las fechas de cruceros que ellos reportan y el área cubierta. Además, los resultados mostrados por Yáñez-Arancibia et al., (1985) y por Yáñez-Arancibia y Sánchez-Gil (1986) fueron obtenidos en la década de los 80 's. Es posible, que después de más de treinta años de diferencia entre esos trabajos y éste, haya existido una variación significativa en la riqueza de especies de la zona, cambiando la cantidad y abundancia de ellas; debido probablemente a efectos tanto de la efectividad del muestreo (uso de embarcación más moderna y con mayor tecnología), del incremento en las actividades pesqueras, como de cambios tanto del clima como de la dinámica del substrato.

En cuanto a la riqueza en el Golfo de México, se tiene el trabajo de HernándezVázquez, (2007) donde reportó una riqueza de 119 especies de larvas de peces en la parte sur del Golfo. El esfuerzo de pesca de HernándezVázquez, (2007) es equiparable al nuestro, pero con la diferencia que sólo fueron colectados peces en estadio larval, pero su riqueza no deja de ser significativa.

Es probable que, si se compara este estudio con otros futuros en épocas climáticas distintas, puedan encontrarse más especies que no fueron reportadas en este listado y quizá corroborar la existencia de las más de 270 especies que mencionan Yáñez-Arancibia y Sánchez-Gil (1986). En otras partes del mundo, Meléndez y Kong (2000) mencionan que existen cerca de 400 especies que habitan a más de 200 $\mathrm{m}$ de profundidad para Chile y para la costa norte del Caribe colombiano, Páramo, GuillotIllidge, Rodríguez y Sánchez-Ramírez (2009) reportan solamente 68 especies no pasando de $100 \mathrm{~m}$ de profundidad.

Haimovici, Silva-Martins, Lima de Figueiredo y Castelli-Viera (1994), hicieron un estudio para peces óseos en la parte sur de Brasil, y encontraron sólo 93 (30 elasmobranquios) a una profundidad de más de $350 \mathrm{~m}$. En nuestro caso, la alta riqueza se debe a los intervalos de profundidades considerados, que fueron desde los escasos $20 \mathrm{~m}$ hasta más de 600. Pequegnat, Gallaway y Pequegnat (1990) mencionan que al interior del Golfo hay un mayor número de especies de profundidad con respecto a las observadas en el océano Atlántico, debido a la presencia de la corriente de lazo sistema de transporte de larvas, peces, plantas y calor a la zona este del Golfo, y el sistema del río Mississippi.

Yáñez-Arancibia et al. (1985) reportaron a nueve especies como las más representativas y/o dominantes en las capturas efectuadas en la Sonda de Campeche. De éstas, hay coincidencias en cinco de ellas con este trabajo. Harengula jaguana Poey, 1865 fue la más importante. Yáñez-Arancibia y Sánchez-Gil (1986) también reportaron a esta especie con parámetros ecológicos dominantes durante todo el año. De la misma manera, García-Abad et al. (1999) confirmaron que es una especie dominante en las comunidades demersales del Golfo y que está bien distribuida en la plataforma continental. Por otro lado, frente a las costas de Veracruz, Castro-González, Silencio-Barrita, Juárez-Silva, Montaño-Benavides y Pérez-Gil (1998) la reportaron como una especie de mediana incidencia en la fauna de acompañamiento del camarón. 
En el presente trabajo esta especie obtuvo un IVI inferior al $1 \%$, debido a que sólo se obtuvieron 25 ejemplares con cerca de un $\mathrm{kg}$ y capturada en únicamente tres estaciones. Cabe mencionar que los estudios sobre H. jaguana, reportan a este cupleido cerca de la línea costera, en isobatas de no más de 30 m (García-Abad et al., 1999).

Polydactylus octonemus (Girard, 1858) y Diplectrum radiale (Quoy \& Gaimard, 1824), especies que también fueron reportadas como dominantes por Yáñez-Arancibia y SánchezGil (1986), se registraron valores con un IVI cercano al $1 \%$. Estas especies no fueron las más dominantes, pero tienen una abundancia, frecuencia y biomasa considerable. Para el caso de Prionotus punctatus también es considerado como dominante; el género Prionotus es de los que presentan mayores abundancias y distribución amplia en la Sonda de Campeche.

En cuanto al resto de las especies, es decir Ariopsis felis, Eucinostomus gula, Synodus foetens, Syacium gunteri y Chloroscombrus chrysurus presentan las biomasas y frecuencias relativas muy similares. En el presente trabajo, se destaca a Micropogonias undulatus, Lutjanus synagris y L. campechanus, Bagre marinus, Conodon nobilis, Stellifer colonensis, Upeneus parvus, Diapterus auratus, D. rhombeus y Narcine brasiliensis como especies dominantes. Para el caso de Lutjanus synagris, Upeneus parvus, Stellifer colonensis y Bagre marinus Yáñez-Arancibia et al. (1985) los consideran dentro de su lista de especies dominantes por abundancia y por peso.

Tal vez, esta variación sea producto de la amplitud de las temporadas y de sitios muestreados. Para D. auratus y D. rhombeus, Aguirre-León y Yáñez-Arancibia (1986) reportaron a estas especies como típicas de ambientes lagunares y estuarinos, aunque no dominantes (Yáñez-Arancibia et al., 1985); los resultados de este trabajo, las señalan como dominantes, posiblemente como resultado de la temporada de muestreo y la eficiencia del mismo.

A pesar que la presencia de $M$. undulatus no fue amplia, lo que la ubicó como especie dominante fue su biomasa, debido a que la mayoría de los ejemplares colectados presentaron tallas de más de $20 \mathrm{~cm}$ con un peso promedio de $381 \mathrm{~g}$; no se colectaron ejemplares juveniles, aun cuando las larvas de esta especie tienden a preferir profundidades bajas (FloresCoto, Figueroa-Páez \& Zavala-Gracía, 1999).

Para la especie Lutjanus campechanus, Yáñez-Arancibia et al. (1985), reportaron 130 ejemplares; en nuestro trabajo su abundancia fue alta (495 ejemplares). Yáñez-Arancibia et al. (1985), reportaron las abundancias de Narcine brasiliensis y de Conodon nobilis muy bajas, comparándose con los datos obtenidos en este trabajo.

Entre las particularidades que posee la fauna demersal, es su uso como elementos importantes de monitoreo del ambiente en que habitan, sobre todo cuando existen actividades o eventos que modifican su estructura (Prena, 1995; Santos, Castel \& Souza-Santos, 1996; Wright, Setzler-Hamilton, Magee, Kennedy \& McIninch, 1996; González-Solis \& Torruco, 2001). Esta fauna permite obtener una visión clara y objetiva de los cambios en el ambiente y sus posibles causas en corto tiempo y a costos razonables (Engle, Summers, \& Gaston, 1994; Weisberg, Ranasinghe, Dauer, Shaffner, \& Frithsen, 1997; González-Solis \& Torruco, 2001; Abarca-Arenas, Franco-López, ChávezLópez, \& Morán-Silva, 2003).

Grall y Glénmerec (1997), mencionan sobre el empleo de la fauna bento-demersal como un modelo de bioindicador basado en el reconocimiento del espectro de la fauna dentro de cada tipo de comunidad; así, los cambios en las características del sedimento como contenido de arena o de materia orgánica, pueden influenciar la epifauna a través de un gradiente (Flint \& Kalke, 1986; Manino \& Montagna, 1997). Es muy probable que los patrones observados en la fauna muestreada en esta investigación, sean influenciados por fuentes puntuales de variación espacial en factores ambientales como la salinidad y las características de los sedimentos. El aporte de agua de los ríos, modifica el contenido de arena y materia orgánica, y genera una variación gradual a lo largo de las desembocaduras. En este trabajo se 
reportan nódulos densos en los atributos comunitarios de los peces en las desembocaduras de los ríos, que poco a poco se van diluyendo conforme se alejan de la costa.

Algunas de las distribuciones identificadas son originadas probablemente por las condiciones para crianza y crecimiento que representan estos ecosistemas para esta fauna. La zona de plataformas, al presentar un nódulo de densidad intermedia (cercano a los 2000 individuos); es probable se deba al refugio que proporcionan esas áreas y a la prohibición de pesca, las especies tienen la posibilidad de proliferar y permite un mayor crecimiento de los organismos. Esta área se prolonga hasta la zona arrecifal de Cayo Arcas, donde la misma protección del arrecife produce un efecto semejante, ya que aquí se presenta la heterogeneidad espacial de la estructura arrecifal, que permite una mayor protección a los organismos, al facilitar zonas de refugio y de alimento. La zona oceánica presenta valores menores, lo cual manifiesta que en esta área solo algunas de las especies son las que aprovechan los recursos disponibles. No obstante, el patrón de la diversidad no muestra un gradiente en todas las áreas, ya que muestra una intrusión de bajas diversidades en áreas con mayor actividad petrolera.

Por otro lado, en algunas regiones del Golfo de México, existe una alternancia de especies y cambios en los ensamblajes de ellas, que no es posible detectarlas ni siquiera anualmente (Torruco, 1988), porque su cambio es muy sutil, ya que presentan ciclos bianuales (Torruco, Chávez \& González, 1990). Con las nuevas herramientas estadísticas, las descripciones y el análisis de los ensamblajes de esta fauna, es posible caracterizar las condiciones, no solo a una escala local, sino también como indicadores potenciales a escalas regionales, lo que permite reflejar y discernir la variación natural de la inducida, aun cuando sean comunidades con una amplia heterogeneidad (Cota \& Santiago, 1992; Weisberg et al., 1997).

En lo que se refiere al uso de las especies como biomonitores, organismos centinelas o bioindicadores; de ellas se ha mencionado que deben tener ciertas características tales como: fácil identificación, sedentarias, abundantes, período de vida prolongada, disponible para muestreos en todo el año, etc. Sin embargo, no todos los organismos marinos cumplen este criterio, de hecho, ni las especies empleadas como biomonitores lo satisfacen totalmente (Rainbow, 1995). Esto implica que, un solo indicador no aporta información suficiente para monitorear ambientes marinos costeros, dada la alta variación en sus condiciones naturales (Schlekat et al., 1994). Una alternativa, sería el uso de índices bióticos, como el presentado por Grall y Glénmarec (1997), donde definen un modelo basado en el reconocimiento de cinco grupos de especies que presentan un perfil de abundancia similar a lo largo de un gradiente de enriquecimiento del ambiente por materia orgánica.

Engle et al. (1994), utilizó un índice béntico para determinar las condiciones de estuarios del Golfo de México, y determinar hábitats degradados y no degradados, para lo cual tomó en cuenta, un índice de diversidad y dos grupos de organismos indicadores, después hacer pruebas de discriminantes canónicos para escoger tanto los organismos indicadores como la medida béntica. En esta área del Golfo de México, se tiene una confluencia de dos substratos: uno de naturaleza kárstica y otro de naturaleza limo-arcillosa, los cual hace difícil la utilización de un índice de estas características. Es por ello que se propone el uso de especies discriminantes para caracterizar cada sitio, sería deseable que se tuvieran las especies en un espectro confiable en la variable tiempo además del espacio con lo cual se robustecerían las conclusiones, pero aún con esta limitación, es posible definir una estructura natural. Bajo este contexto, las 24 especies identificadas serían las que deben ser monitoreadas frecuentemente, ya que en ellas reside la síntesis de las características totales de cada una de las estaciones que ellas discriminan, de ahí su importancia.

En relación a las interacciones entre los descriptores comunitarios y los parámetros del ambiente, el cuadro 5 muestra la presencia de la variable Carbono Orgánico como significativa, 
tanto para la abundancia como para la biomasa, y del REDOX para la riqueza de especies. Esto muestra un efecto directo entre el factor ambiental y el macrodescriptor, que puede ser evidenciado por la alta varianza explicada en el primer componente del ACP; no obstante, algunas variables pudieran tener altas relaciones secundarias entre sí y probablemente puedan acoplar sus efectos sobre la ictiofauna.

Un aspecto que resalta, es la relación que guarda la profundidad con estos descriptores, que aun cuando no son significativos, si influyen de manera negativa, denotando una variación espacial de la comunidad de los peces demersales del Banco de Campeche, conformada por infracomunidades que presentan características propias de cada estrato que en este caso se identifican dos; por un lado el estrato formado por peces de aguas poco profundas y por el estrato profundo (Flores et al., 1999), en trabajos similares, varios autores han encontrado una estratificación, en relación con la profundidad, en la distribución de especies de peces, distinguiendo diferentes gradientes estructurales en la comunidad (Jongman, Ter Baak, \& Van Tongeren, 1987; Qiu, 1988; Fujita, Inada, \& Ishito, 1995; Massuti, Renones, Carbonell, \& Oliver, 1996).

Otros estudios han demostrado que los cambios en la ictiofauna están influenciados por las variaciones de los parámetros físicoquímicos, como la temperatura y salinidad o la disponibilidad de nutrientes, que están estrechamente relacionados con la profundidad, así como a diferentes tipos de sedimento (Sánchez, 1993). Este trabajo tiene una amplia variedad de profundidades y tipos de substratos, que se arreglan como un gran mosaico de hábitats, $\mathrm{Si}$ bien la fauna ictica realiza migraciones alimenticias a lo largo de la columna de agua (Sharp, 1988), en este caso con ese mosaico de hábitats bénticos, también realiza migraciones horizontales para la búsqueda de alimento y su posterior regreso a áreas de refugio. Esto es posible vislumbrarlo en la distribución espacial.

De la relación obtenida entre los factores del ambiente con los macrodescriptores comunitarios, se infiere que aun cuando existan contaminantes derivados de hidrocarburos o plaguicidas, el efecto de estos es menos evidente que el efecto de las interacciones ecológicas, donde los procesos de competencia por recursos, depredación y preferencias de hábitats, siguen siendo para esta fracción, muy importantes, ya que direccionan la estructura de la comunidad bentodemersal. Aun cuando el desarrollo de las actividades de la industria petrolera y pesquera originen un impacto de deterioro a largo plazo sobre las comunidades de la plataforma continental (Flint, 1981; Flint \& Rabalais, 1981), este no se muestra con facilidad. Por lo tanto, se deberán de establecer estudios de campo acotados para establecer las fluctuaciones naturales de los organismos clave para diferenciarlas de las provocadas por las actividades petroleras, esto conlleva a que es necesario tener el componente tiempo en esas fluctuaciones naturales, que con este tipo de análisis no se tiene.

De lo anterior se puede concluir lo siguiente: (1) Inicialmente se debe limitar la magnitud del estudio y adaptar la estrategia de acuerdo a ello. Si este es a un nivel local y en ambientes con poca variación en sus parámetros fisicoquímicos, es posible utilizar solo una especie como indicador de las alteraciones ambientales, sin embargo, esta estrategia no debe ser aplicada en zonas con una amplia variación en sus parámetros y diversidad de hábitats, o en estudios de escala regional. En estos casos, es recomendable aplicar otra metodología en donde se involucren varias medidas bénticas y diversas especies, a fin de cubrir un espectro amplio dentro de las comunidades. (2) La selección y utilización de las especies dependerá también de qué tanto reflejen las alteraciones de los elementos que se quieran conocer, ya sean derivadas por algún perturbador ambiental o por la dinámica propia del sistema.

\section{RESUMEN}

La sonda de Campeche posee gran diversidad de especies, heterogeneidad de hábitats y fuerte incidencia de las industrias petrolera y pesquera. El objetivo de este trabajo fue obtener la distribución espacial de la ictiofauna y su relación con elementos del ambiente, así como 
implementar una línea base que sirva como referente para futuras contingencias que puedan ocasionar estas industrias. Se empleó una base de datos obtenida en 2010, con 54 localidades en la Sonda de Campeche que fueron muestreadas mediante arrastres camaroneros. Se colectaron $2502438 \mathrm{~kg}$ de material biológico y 33315 peces de 2 clases (Chondrichthyes y Actinopterygii) con un total de 193 especies. De ellas, 17 fueron dominantes, asociadas a fondos arenosos y lodosos, aunque también hubo especies de ambientes estuarinos, de arrecife y de hábitos pelágicos. La diversidad $\left(H^{\prime}\right)$ promedio fue de 3.2 bits/individuos. Las familias más comunes fueron los Perciformes (84 species), Pleuronectiformes (23), Scorpaeniformes (15) and Tetraodontiformes (14); las riquezas de especies más altas fueron: Sciaenidae (15 especies), Paralichthyidae (12), Carangidae (10), y Triglidae y Synodontidae (7). Los géneros más comunes fueron: Prionotus (6 especies), Citharichthys and Sphoeroides (4 cada uno). Por cada localidad, la riqueza fluctuó entre 3 y 6 especies con un promedio de 46 $\mathrm{kg}$. La composición de especies fue diferente entre hábitats y áreas. La ordenación comunitaria presenta una concordancia al identificar espacialmente cada zona. El análisis paisajístico define nódulos de alta densidad y diversidad que corresponden con áreas de desembocaduras de ríos, lagunas y de zonas arrecifales. De las relaciones entre las variables y los descriptores comunitarios, sólo el Carbono Orgánico fue significativo, tanto para la abundancia como para la biomasa, el REDOX para la riqueza de especies y las otras variables no fueron importantes.

Palabras clave: ictiofauna; Sonda de Campeche; Golfo de México; arrastres camaroneros.

\section{REFERENCIAS}

Abarca-Arenas, L. G., Franco-López, J., Chávez-López, R., \& Morán-Silva, A. (2003). Estructura de la comunidad de peces de la pesca incidental camaronera. In A. Wakida-Kusunoki, R. Solana-Sansores, \& J. A. Uribe-Martínez (Eds.), Memorias del III Foro de Camarón del Golfo de México y del Mar Caribe (pp.70-74). México: INP-SAGARPA.

Aguirre-León, A., \& Yáñez-Arancibia, A. (1986). Las mojarras de la laguna de Términos: taxonomía, biología, ecología y dinámica trófica (Pisces: Gerreidae). Anales del Instituto de Ciencias del Mar y Limnología, UNAM, 13(1), 369-444.

Brassard, P. (1997). Measurement of $\mathrm{Eh}$ and $\mathrm{pH}$ in aquatic sediments. In A. Mudroch, J. M. Azcue, \& P. Mudroch (Eds.), Manual of physico-chemical analysis of aquatic sediments (pp. 47-69). New York, USA: CRC Press, Inc. Lewis Publishers.

Bricker, O. P. (1982). Redox potential: Its measurement and importance in water systems. In R. A. Minear, \& L. H. Keith (Eds.), Water analysis. Vol 1: Inorganic species (pp. 55-79). Florida, USA: Academic Press.
Buchanan, J. B. (1984). Sediment Analysis. In N. A. Holme, \& A. D. Mc Intyre (Eds.), Methods for the study of marine benthos (pp. 41-65). Oxford: Blackwell Scientific Publications.

Bumes-Romo, R. (2009). Estatus taxonómico de Gerres cinereus (Walbaum, 1972) (Teleostei: Gerreidae). (Tesis Maestría). CICIMAR-IPN, La Paz B.C.S., México.

Carpenter, K. E. (2002a). FAO Species identification field guide for fishery purpose. The living marine resources of Western Central Atlantic. Vol. 1: Introduction, mollusk, crustaceans, hagfishes, shark, batoid fishes and Chimeras. Rome: FAO.

Carpenter, K. E. (2002b). FAO Species identification field guide for fishery purpose. The living marine resources of the Western Central Atlantic. Vol. 2: Bony fishes part 1 (Acipenseridae to Grammatidae). Rome: FAO.

Carpenter, K. E. (2002c). FAO Species identification field guide for fishery purpose. The living marine resources of the Western Central Atlantic. Vol. 3: Bony fishes part 2 (Opistognathida to Molidae), sea turtles and marine mammals. Rome: FAO.

Castro-Aguirre, J. L., \& Espinoza-Pérez, H. (1996). Listado faunístico de Mexico. VII Catálogo sistemático de las rayas y especies afines de México: Chondrichthyes: Elasmobranchi: Rajiformes: Batoideiomorpha. UNAM, México.

Castro-González, M. I., Silencio-Barrita, J. L., JuárezSilva, M. E., Montaño-Benavides, S., \& Pérez-Gil, F. (1998). Composición química de la fauna de acompañamiento del camarón de Veracruz (Golfo de México). Revista de Biología Tropical, 6(2), 249-256.

Cota, F. V., \& Santiago, R. (1992). Estudio de la estructura de las comunidades de peces de la laguna de Tampamachoco, Ver. IX Congreso Nacional de Oceanografia, Veracruz, México.

Dickson, H., \& Moore, R. (1977). Fishes of the Gulf of Mexico, Texas, Louisiana, and adjacent waters. EUA: Texas A\&M University Press.

Engle, D. V., Summers, K. J., \& Gaston, R.G. (1994). A benthic index of environmental condition of Gulf of Mexico estuaries. Estuaries, 17(2), 372-384.

Ezcurra, E., \& M. Equihua. (1984). La teoría de la información aplicada a la clasificación de datos biológicos. In E. Ezcurra, M. Equihua, B. Kohlmann, \& S. SánchezColón (Eds). Métodos cuantitativos en la biogeografia (pp. 9-40). CDMX: Instituto de Ecología A.C.

Flint, R. W. (1981). Gulf of Mexico outer continental shelf benthos: macroinfaunal environmental relationships. Biology and Oceanography, 1,135-145. 
Flint, R. W., \& Rabalais, N. (1981). Environmental studies of a marine system: South Texas outer continental shelf. Texas: Texas University Press.

Flint, R. W., \& Kalke, R. D. (1986). Niche characterization of dominant estuarine benthic species. Estuarine Coastal Shelf Science, 22, 657-674.

Flores-Coto, C., Figueroa-Páez, P., \& Zavala-Gracía, F. (1999). Distribución y abundancia de larvas de Scianidae en la columna de agua, en el sur del Golfo de México: primavera. Hidrobiología, 9(2), 135-144.

Fujita, T., Inada, T., \& Ishito, Y. (1995). Depth-gradient structure of the demersal fish community on the continental shelf and upper slope off Sendai Bay, Japan. Marine Ecology Progress Series, 118(1-3), 13-23.

García-Abad, M. C., Tapia-Gracía, M., Yañez-Arancibia, A., \& Sánchez-Gil, P. (1999). Distribución, abundancia y reproducción de Harengula jaguana Goode y Bean 1879, en la plataforma continental del sur del Golfo de México (Pisces: Clupeidae). Biotrópica 31(3), 498-501.

García-Cuellar, J. A., Arreguín-Sánchez, F., Hernández, S. V., \& Lluch-Cota, B. (2004). Impacto ecológico de la industria petrolera en la Sonda de Campeche, México, tras tres décadas de actividad: una revisión. Interciencia, 29(6), 311-319.

González-Solis, M. A., \& Torruco, D. (2001). La fauna béntica del estero de Sabancuy, Campeche, México. Revista de Biología Tropical, 49(1), 31-45.

Grall, J., \& Glénmerec, M. (1997). Using biotic indices to estimate macro benthic community perturbations in the Bay of Brest. Estuarine Coastal and Shelf Science, 44 (Suplement A), 43-53.

Haimovici, M., Silva-Martins, A., Lima de Figueiredo J., \& Castelli-Viera, P. (1994). Demersal bony fish of the outer shelf and upper slope of the southerns Brazil subtropical convergence ecosystem. Marine Ecology Progress Series, 108, 59-77.

Hernández-Vázquez, T. P. (2007). Caracterización espacial de la comunidad de larvas de peces y su relación con variables ambientales y de contaminación en el sur del Golfo de México. (Tesis de Maestría). Mérida Yucatán, México: CINVESTAV-IPN.

Jongman, R. H., Ter Baak, C. J. M., \& Van Tongeren, O. F. R. (1987). Data analysis in community and landscape ecology. PUDOC, Wageninger, Netherlands.

Lavett, C. (1997). Field guide to tropical marine fishes of the Caribbean, the Gulf of Mexico, Florida, The Bahamas and Bermuda. USA: National Audubon Society.

Lozano-Villano, M. de L., García-Ramírez, M. E., \& Contreras-Balderas, S. (1993). Peces costeros y marinos del Estado de Veracruz. In S. I. Salazar-Vallejo,
\& N. E. González (Eds), Biodiversidad Marina y Costera de México (576-595 pp.). México: Comisión Nacional para el Conocimiento y Aprovechamiento de la Biodiversidad (CONABIO) y Centro de Investigación de Quintana Roo (CIQRO).

MacEachran, J. D., \& Fechhelm, J. D. (1988a). Fishes of the Gulf of Mexico: Vol 1. EUA: University of Texas Press.

MacEachran, J. D., \& Fechhelm, J. D. (1988b). Fishes of the Gulf of Mexico: Vol 2. EUA: University of Texas Press.

Manino, A., \& Montagna, A. P. (1997). Small-scale spatial variation of macrobenthic community structure. Estuaries, 20(1), 159-173.

Massuti, E., Renones, O., Carbonell, A., \& Oliver, P. (1996). Demersal fish communities exploited on the contionental shelf and slope off Majorca (Balearic Islands, NW Mediterranean). Vie-Milieu, 46(1), 45-55.

Meléndez, R., \& Kong, I. U. (2000). Sistemática filogenética en peces de ambientes profundos presentes en Chile. Estudios Oceanológicos, 19, 129-136.

Nelson, J. (1994). Fishes of the world. 3rd edition. New Jersey, EUA: John Wiley \& Sons, Inc. Hoboken.

Nelson, J. (2006). Fishes of the world. 4th edition. New Jersey, EUA: John Wiley \& Sons, Inc. Hoboken.

Orlocí, L. (1978). Multivariate analysis in vegetation research. Netherlands: Springer Netherlands.

Páramo, J., Guillot-Illidge, L., Rodríguez, A., \& SánchezRamírez, C. (2009). Aspectos poblacionales y ecológicos de peces demersales de la zona norte del Caribe colombiano en relación con el hábitat: una herramienta para identificar áreas marinas protegidas (AMPs) para el manejo pesquero. Caldasia, 31(1), 123-144.

Pequegnat, W. E., Gallaway, B. J., \& Pequegnat, L. H. (1990). Aspects of the ecology

of the deep-water fauna of the Gulf of Mexico. American Zoology, 30, 45-64.

Pielou, E. C. (1977). Mathematical ecology. 2nd edition. New York: John Wiley and Sons.

Powell, S. M., Headrich, R. L., \& McEachran, J. D. (2003). The deep-sea demersal fish fauna of the northern Gulf of Mexico. Journal of Northwest Atlantic Fishery Science, 31, 19-33.

Prena, J. (1995). Temporal irregularities in the macrobenthic community and deep-water advection in Wismar Bay (western Baltic Sea). Estuarine Coastal Shelf Science, 41,705-717. 
PNUMA (Programa de las Naciones Unidas). (2003). GEO América Latina y el Caribe, perspectivas del medio ambiente 2003: Cap. 2 Estado de medio ambiente en América Latina y el Caribe 1972-2002. México: ORPALC, PNUMA.

Qiu, Y. (1988). The regional changes of fish community on the northern continental shelf of South China Sea. Journal Fish China Shuichan Xuebao, 12(4), 303-314.

Rainbow, S. P. (1995). Biomonitoring of heavy metals availability in the marine environment. Marine pollution bulletin, 31(4-12), 183-192.

Rossi, R. E., Mulla, D. J., Joumel, A. G., \& Franz, E. H (1992). Geostatistical tool for modeling and interpreting ecological spatial dependence. Ecological monographs, 65(3), 235-260.

Sánchez, F. (1993). Las comunidades de peces de la plataforma del Cantábrico. Publ. Especiales. España: Instituto Español de Oceanografía.

Sánchez-Gil, P., Yáñez-Arancibia, A., \& Amezcua-Linares, F. (1981). Diversidad, distribución y abundancia de las especies y poblaciones de peces demersales de la Sonda de Campeche (verano 1978). Anales del Instituto de Ciencias del Mar y Limnología, 8(1), 209-240.

Santos, P. J. P., Castel, J., \& Souza-Santos, P. L. (1996) Seasonal variability of meiofaunal abundance in the oligo-mesohaline area of the Gironde Estuary, France. Estuarine, Coastal and Shelf Science, 43, 549-563.

Schlekat, C. E., McGee, B. I., Boward, D. M., Reinharz, E., Velinsky, D. J., \& Wade, T. L. (1994). Tidal river sediments in the Washington D.C. area. III. Biological effects associate with sediment contamination. Estuaries, 17(2), 334-344.

Sharp, G. D. (1988). Fish population and fisheries, their perturbations, natural and man-induced. In H. Postma, \& J. J. Zijlstra (Eds.), Continental Shelves (pp. 155-202). New York: Elsevier.
Torres, R. E., \& Orozco, B. (1991). Los peces de México1a edición. México: AGT Editor.

Torruco, D. (1988). Estructura de la fauna nectobentónica del sublitoral de Laguna Verde, Veracruz. (Tesis de maestría). Mérida Yucatán, México: Centro de Investigación y de Estudios Avanzados del IPN, Unidad Mérida.

Torruco, D., Chávez, E. A., \& González, M. A. (1990). Spatio-temporal variation of the structure organization of demersal communities in the southwestern Gulf of Mexico. Revista de Biología Tropical, 55(2), 509-536.

Weisberg, B. S., Ranasinghe, A. J., Dauer, M. D., Shaffner, C. L., \& Frithsen, B. J. (1997). An estuarine benthic index of biotic integrity (B-IBI) for Chesapeake Bay. Estuaries, 20(1), 149-158.

Wright, A. D., Setzler-Hamilton, M. E., Magee, A. J., Kennedy, S.V., \& McIninch, P. S. (1996). Effect of salinity and temperature survival and development of young Zebra (Dreissena polymorpha) and Quagga (Dreissena bugensis) mussels. Estuaries, 19(3), 619-628.

Yáñez-Arancibia, A., Lara-Domínguez, A.L., Sánchez-Gil, P., Vargas-Maldonado, I., García-Abad, M.C., Álvarez-Guillen, H., Tapia-García, M., Flores-Hernández, D., \& Amezcua-Linares F. (1985). Ecology and evaluation of fish community in coastal ecosystem: Estuary-Shelf relationships in the southern Gulf of Mexico. Chap. 22. In A. Yáñez-Arancibia (Ed). Fish community ecology in estuaries and coastal lagoons: Towards and ecosystem integration. México: UNAM Press.

Yañez-Arancibia, A., \& Sánchez-Gil, P. (1986). Los peces demersales de la plataforma continental del sur del Golfo de México. 1. Caracterización ambiental y ecológica y evaluación de las especies, poblaciones y comunidades. Publicaciones especiales del Instituto de Ciencias del Mar y Limnología, 9, 1-212. 